\title{
An x-ray emission and density functional theory study of the electronic structure of $\mathrm{Zn}_{1-x} \mathrm{Mn}_{x} \mathrm{~S}$
}

\author{
R G Wilks ${ }^{1}$, E Z Kurmaev² ${ }^{2}$ L M Sandratskii ${ }^{3}$, A V Postnikov ${ }^{2}$, \\ L D Finkelstein ${ }^{2}$, T P Surkova ${ }^{2}$, S A Lopez-Rivera ${ }^{4}$ and A Moewes ${ }^{1}$ \\ ${ }^{1}$ Department of Physics and Engineering Physics, University of Saskatchewan, 116 Science Place, \\ Saskatoon, SK, S7N 5E2, Canada \\ 2 Institute of Metal Physics, Russian Academy of Sciences-Ural Division, Yekaterinburg 620219, \\ Russia \\ ${ }^{3}$ Max-Planck Institut für Mikrostrukturphysik, D-06120 Halle, Germany \\ ${ }^{4}$ Laboratorio de Fisica Aplicada, Universidad de los Andes, Merida 5101, Venezuela \\ E-mail: regan.wilks@usask.ca
}

Received 10 July 2006, in final form 14 September 2006

Published 3 November 2006

Online at stacks.iop.org/JPhysCM/18/10405

\begin{abstract}
Mn 3d electronic states in the dilute magnetic semiconductor $\mathrm{Zn}_{1-x} \mathrm{Mn}_{x} \mathrm{~S}$ $(x=0.1-0.3)$ are studied using soft $\mathrm{x}$-ray emission (XES) measurements and density functional theory (DFT). Mn $\mathrm{L}_{2,3}$ emission spectra of $\mathrm{Zn}_{1-x} \mathrm{Mn}_{x} \mathrm{~S}$ ( $x=0.1-0.3$ ) suggest that the Mn impurities do not form clusters in the host $\mathrm{ZnS}$ lattice, in agreement with previous models. A shift in the position of a Mn $\mathrm{L}_{3}$ XES feature suggests a change in the nature of the hybridization between the $\mathrm{Mn} 3 \mathrm{~d}_{3 / 2}$ and S $3 \mathrm{p}$ states as a function of $x$. Our DFT calculations reproduce the weak interatomic exchange interaction, as well as the strong intra-atomic exchange splitting that is expected from observations of Zeeman splitting in such materials.
\end{abstract}

\section{Introduction}

Following the recent discovery of ferromagnetism in ( $\mathrm{Ga}, \mathrm{Mn}$ )As (with a Curie temperature of $110 \mathrm{~K})$ [1, 2] the interest in traditional II-VI semiconductors has been renewed. It has also been suggested that II-VI dilute magnetic semiconductors (DMS) can be used as a source of spin-polarized charge carriers in spin-injection experiments [3]. No magnetic ordering has been experimentally detected down to very low temperatures in $\mathrm{Zn}_{1-x} \mathrm{Mn}_{x}(\mathrm{~S} / \mathrm{Se})$ samples with low Mn concentration [4]. However, the application of a magnetic field leads to giant Zeeman splitting at the top of the valence band. The origin of this splitting is a strong exchange interaction between Mn $3 d$ states and the $p$ states of the semiconductor ( $p-d$ exchange). The combination of strong $\mathrm{p}-\mathrm{d}$ exchange interaction and very weak $\mathrm{Mn}-\mathrm{Mn}$ exchange interaction is an interesting feature of this class of DMS. Theoretical studies of II-VI-type DMS based on 
a model Hamiltonian approach have shown that model parameters can be adjusted to reproduce experimental determinations of exchange interactions $[5,6]$.

However, the number of parameter-free density functional theory (DFT) calculations of the exchange interactions in II-VI dilute magnetic semiconductors is still very small [7]. Recently, it has been shown [8] that the DFT calculations for large supercells of semiconductor crystals can describe the magnetic properties of both III-V and II-VI DMS. The character of the states at the top of the valence band is of critical importance for any potential magnetic or spintronic applications of the III-V and II-VI DMS. In particular, the energy position of the Mn $3 \mathrm{~d}$ states and the strength of their hybridization with the semiconductor states are of major interest and still issues of much debate and controversy—see for example a recent paper by Rader et al [9] and references therein.

A number of spectroscopic studies of dilute metal semiconductors have been performed in recent years. Confining the discussion to studies of compounds of the form $\mathrm{Zn}_{1-x} \mathrm{Mn}_{x} \mathrm{Y}$ (where $\mathrm{Y}$ can be $\mathrm{O}, \mathrm{S}, \mathrm{Se}$, or Te), it is clear that the Mn 2p XAS in these compounds has, in particular, been well studied [10-14]. Other studies have focused on the 1s or $2 p$ absorption spectra of the matrix elements $\mathrm{Zn}$ and $\mathrm{S}[11,15,16]$. Schuler et al recently presented a comparison of the resonant and nonresonant $\mathrm{Mn} \mathrm{L}_{2,3}$ emission spectra of $\mathrm{Zn}_{0.9} \mathrm{Mn}_{0.1} \mathrm{~S}$ to DFT-based density of states (DOS) calculations. In this paper, we present a similar study of the nonresonant Mn $\mathrm{L}_{2,3}$ XES, expanded to include the $\mathrm{S}_{2,3}$ XES measurements. The focus of the current study is the evolution of the Mn-S hybridization as a function of Mn concentration.

\section{Experimental and calculation details}

Using synchrotron radiation from Beamline 8.0.1 of the Advanced Light Source at Lawrence Berkeley National Laboratory, we have measured nonresonant $\mathrm{Mn} \mathrm{L}_{2,3}(3 \mathrm{~d} 4 \mathrm{~s} \rightarrow 2 \mathrm{p})$ and $\mathrm{S} \mathrm{L}_{2,3}$ $(3 \mathrm{~d} 3 \mathrm{~s} \rightarrow 2 \mathrm{p})$ x-ray emission spectra (XES). The excitation energies for $M n L_{2,3}$ and $\mathrm{S} \mathrm{L}_{2,3}$ emission were 660 and $170 \mathrm{eV}$, respectively, which are well above the manganese and sulphur L edges. The $\mathrm{Mn} \mathrm{L}_{2,3}$ and $\mathrm{S} \mathrm{L}_{2,3}$ XES measurements were recorded with energy resolutions of approximately 0.7 and $0.3 \mathrm{eV}$, respectively. All spectra presented here were measured at room temperature.

The DFT calculations of $\mathrm{Zn}_{1-x} \mathrm{Mn}_{x} \mathrm{~S}(x=0.03125)$ are based on a supercell approach, in which one of $32 \mathrm{Zn}$ atoms in a 64-atom $\mathrm{ZnS}$ supercell is replaced by $\mathrm{Mn}$. The calculations were performed using the augmented spherical waves (ASW) method within the local density approximation (LDA and LDA $+U$ techniques) [8] The cubic supercell has a doubled experimental lattice parameter compared with $\mathrm{ZnS}$ [17]. Octahedral and tetrahedral interstitials in the zinc blende lattice, situated in the conventional (single-cell) setting at $\left(\frac{1}{2} \frac{1}{2} \frac{1}{2}\right)$ and $\left(\frac{3}{4} \frac{3}{4} \frac{3}{4}\right)$, correspondingly, were packed in our calculation with empty spheres. That made a supercell of, in total, 128 centres, with all equal-size (space-filling) atomic spheres.

\section{Results and discussion}

\subsection{Mn $L_{2,3}$ emission spectra}

When Mn atoms are inserted into a $\mathrm{ZnS}$ host lattice, there are three possible types of microstructure that could be formed: (1) Mn atoms substitutionally occupying Zn sites, (2) Mn clusters and (3) Mn-S complexes. Nonresonant $\mathrm{Mn} \mathrm{L}_{2,3}$ XES of $\mathrm{Zn}_{1-x} \mathrm{Mn}_{x} \mathrm{~S}$, Mn metal, and $\mathrm{MnTe}$ are shown in figure 1 . The Mn $3 \mathrm{~d}$ valence states are probed via the $3 \mathrm{~d} \rightarrow 2 \mathrm{p}_{1 / 2,3 / 2}$ emission transitions. The intensity ratio $I\left(\mathrm{~L}_{2}\right) / I\left(\mathrm{~L}_{3}\right)$ is rather small for the pure metal, due to the Coster-Kronig $(\mathrm{C}-\mathrm{K})$ process $\mathrm{L}_{2} \mathrm{~L}_{3} \mathrm{M}_{4,5}$. It is known that the $I\left(\mathrm{~L}_{2}\right) / I\left(\mathrm{~L}_{3}\right)$ intensity 


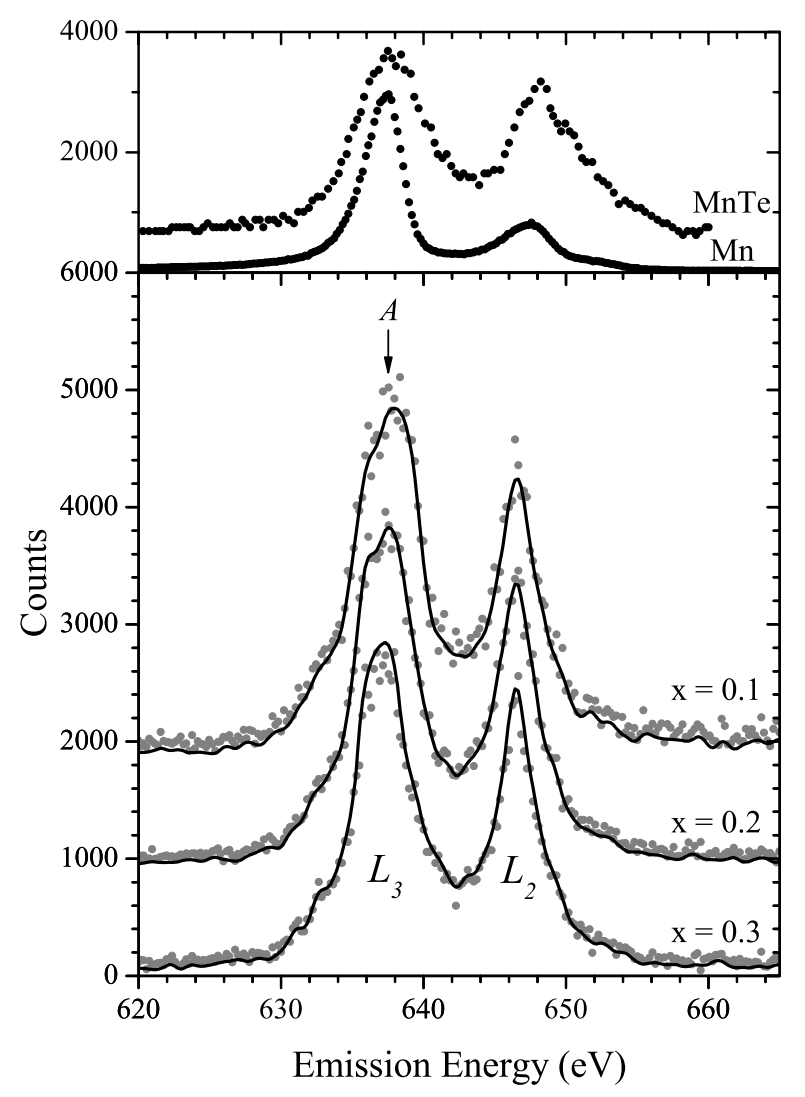

Figure 1. Nonresonant $\mathrm{Mn} \mathrm{L}_{2,3}$ XES of $\mathrm{Zn}_{1-x} \mathrm{Mn}_{x} \mathrm{~S}(x=0.1-0.3)$ and reference samples of MnTe and $\mathrm{Mn}$ metal. The raw data for the $\mathrm{Zn}_{1-x} \mathrm{Mn}_{x} \mathrm{~S}$ spectra are shown as grey dots in the upper panel, with the black line representing the result of a FFT smoothing algorithm applied using Origin 7.0.

ratio for $3 \mathrm{~d}$ metals becomes larger with increasing oxidation. This effect is due to the fact that the nonradiative $\mathrm{L}_{2} \mathrm{~L}_{3} \mathrm{M}_{4,5} \mathrm{C}-\mathrm{K}$ transition probability is expected to be lower for $3 \mathrm{~d}$ metal compounds than for the pure metal. It has been proposed that in insulators, only the $\mathrm{C}-\mathrm{K}$ transition with excitation of individual atoms is allowed, whereas in metals a new type of $\mathrm{C}-\mathrm{K}$ transition is realized, in which energy is dissipated through collective excitations of electron density vibrations $[18,19]$. Such excitations are most efficient at $\sim 15-20 \mathrm{eV}$, which neatly coincides with the $2 \mathrm{p}$ spin-orbit splitting. The intensity ratio $I\left(\mathrm{~L}_{2}\right) / I\left(\mathrm{~L}_{3}\right)$ of $\mathrm{Mn}$-doped $\mathrm{ZnS}$ in figure 1 is close to that of insulating MnTe and differs significantly from that of the Mn metal. This observation rules out a clustering of Mn impurities in $\mathrm{Zn}_{1-x} \mathrm{Mn}_{x} \mathrm{~S}$, in agreement with the conclusions drawn from the EXAFS studies of $\mathrm{Zn}_{1-x} \mathrm{Mn}_{x}$ Se [20].

In addition to the information provided by the $I\left(\mathrm{~L}_{2}\right) / I\left(\mathrm{~L}_{3}\right)$ ratio, close examination of the shapes of the $L_{3}$ features suggests a variation in the electronic structure of the Mn valence states as a function of the Mn concentration. The feature labelled $A$ (approximately $637.6 \mathrm{eV}$ ) in figure 1 shifts to lower energy as a function of $x$. The main peak shifts from $637.9 \mathrm{eV}$ $(x=0.1)$, to $637.6 \mathrm{eV}(x=0.2)$, to $637.3 \mathrm{eV}(x=0.1)$, for a total shift of $0.6 \mathrm{eV}$. We propose that this feature is associated with $\mathrm{Mn} 3 \mathrm{~d}_{5 / 2}$ states that have been partially filled as a result of the interaction with the $\mathrm{S} 3 \mathrm{p}$ electrons. This suggestion is supported by the fact that the shape of the $L_{2}$ feature, which is produced only by $3 d_{3 / 2} \rightarrow 2 p_{1 / 2}$ transitions, remains 


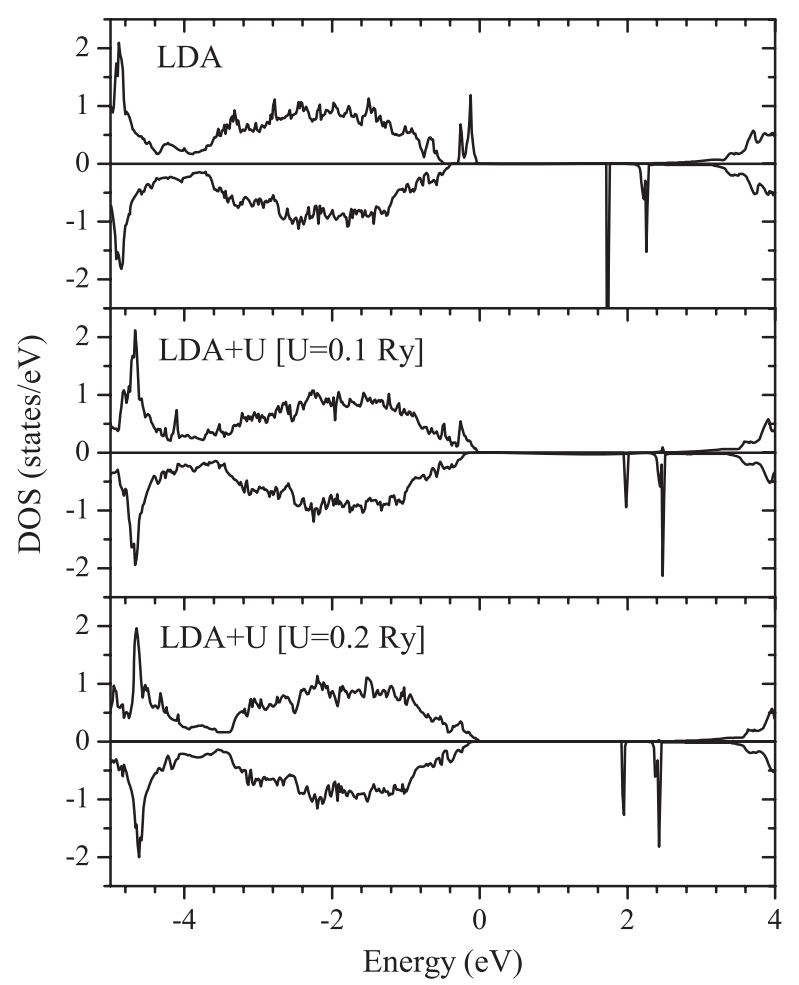

Figure 2. Spin-resolved total DOS of $\mathrm{Zn}_{1-x} \mathrm{Mn}_{x} \mathrm{~S}(x=0.03125)$ with $U=0,0.1$, and 0.2 Ryd. The 'zero' energy represents the Fermi level.

unchanged. It is likely that the effect observed in the $\mathrm{Mn} \mathrm{L}_{3}$ XES is related to previously reported changes in the shape of the S 1s XAS spectra of similar materials [16]. In the study by Pong et al, variations in the XAS spectra were attributed to the increasing likelihood of $S$ 3p/Mn 3d hybridization as the concentration of the Mn was increased. The observed features were specifically attributed to transitions into the unoccupied $\mathrm{S} 3 \mathrm{p} / \mathrm{Mn} 3 \mathrm{~d}\left(\mathrm{t}_{2}\right)$ levels. Our results suggest that we have directly observed the related change in the analogous occupied levels of $\mathrm{Zn}_{1-x} \mathrm{Mn}_{x} \mathrm{~S}$. As the concentration of Mn increases, the hybrid Mn $3 \mathrm{~d}_{5 / 2} / \mathrm{S} 3 \mathrm{p}$ orbitals become more Mn-like, approaching degeneracy with the metallic $3 \mathrm{~d}_{3 / 2}$ states. This shift in energy is similar to a Mn concentration-dependent opening of the band gap that was observed in some Mn-doped $\mathrm{ZnS}$ nanoclusters [21], although, unlike the band gap study, we observe no saturation of this effect in our spectra.

\subsection{LDA calculations}

Our supercell included one Mn atom, which implied ferromagnetic interaction with its repeated replicas at the distances of the supercell edge. Because of the large separation, the Mn $3 \mathrm{~d}$ states behave like impurity levels in a semiconductor band gap (top panel of figure 2). Due to strong intra-atomic exchange splitting on the Mn site, its five d states, all with the same spin direction, become occupied, just above the top of the semiconductor valence band. In the other spin channel, the vacant states are formed just below the conduction band bottom. The doped material remains semiconducting, thanks to the full magnetization of the impurity $3 \mathrm{~d}$ states obtained by replacing $\mathrm{Zn}$ with $\mathrm{Mn}$ : the supercell loses exactly five electrons in one spin 


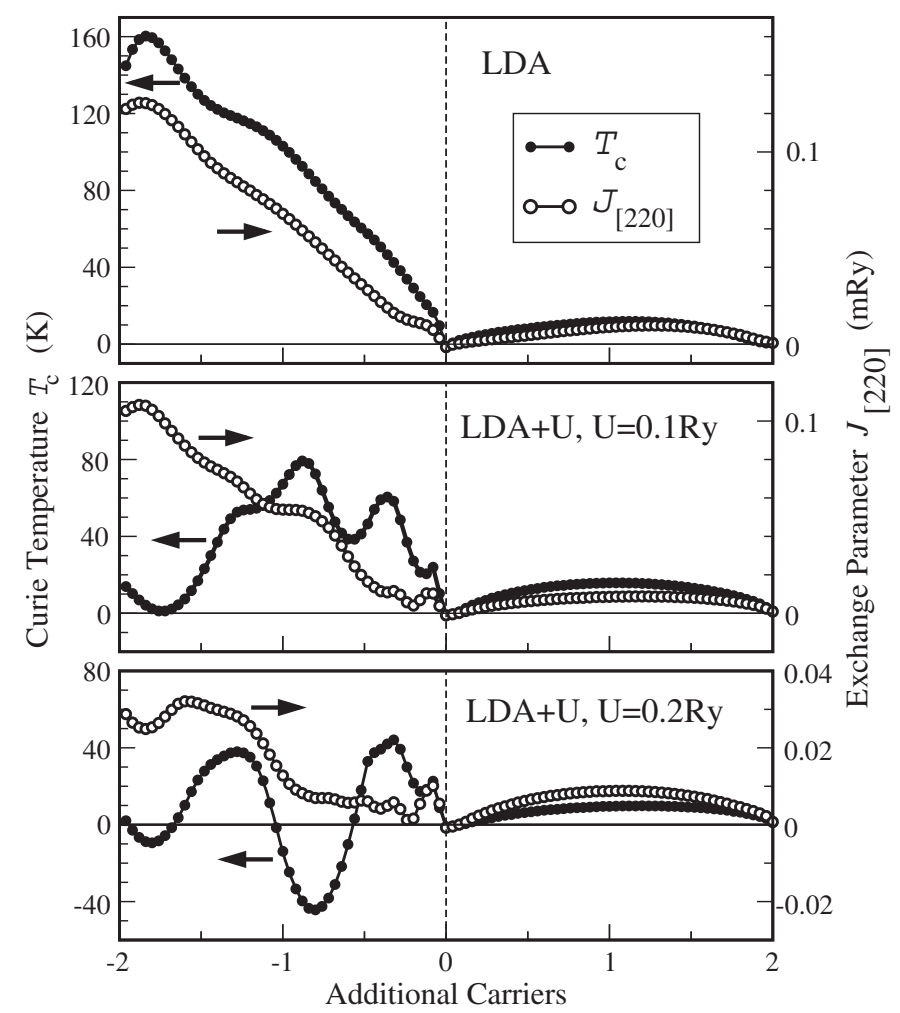

Figure 3. The calculated Curie temperature and leading Heisenberg exchange parameter as a function of the band occupation with $U=0,0.1$, and 0.2 Ryd. The nominal number of electrons corresponds to the zero value at the abscissa axis.

channel. However, due to the hybridization involved, the resulting magnetic moment is not fully localized and amounts only to $4.33 \mu_{\mathrm{B}}$ within the Mn atomic sphere (the number of Mn $3 \mathrm{~d}$ electrons is 5.35).

Large Mn atomic moments and the strong exchange interaction between Mn $3 \mathrm{~d}$ states and states of the semiconducting matrix raise the question of the physical reason for the absence of magnetic ordering in the system up to very low temperatures. To address this question, we performed a calculation of the parameters of Heisenberg's exchange interactions between the Mn moments. The calculations were performed within the parameter-free DFT scheme based on the frozen-magnon approach as described in [6].

The top panel of figure 3 shows the calculated leading exchange parameter $J_{[220]}$, between the pair of Mn atoms separated by the vector $a(220)$, as well as the Curie temperature estimated within the mean-field approximation $k_{\mathrm{B}} T_{\mathrm{C}}=\frac{2}{3} \sum_{n} J_{n}$. Here $a$ is the lattice parameter of the semiconductor matrix and $J_{n}$ is the set of interatomic exchange parameters. Negative values of the Curie temperature in this plot correspond to negative values of the exchange parameters and reveal instability of the ferromagnetic state. To gain deeper insight into the formation of the interatomic exchange parameters, we performed the calculation allowing variable electron numbers. The $n=0$ value corresponds to the nominal band occupation with the valence band filled and the conduction band and the impurity spin-down bands empty. The tiny value that is found for $J$ is consistent with the failure to detect magnetic ordering experimentally. The small values of the interatomic exchange interactions are due to two factors [22]. The first is a large 
semiconducting gap that makes the contribution of the empty states to the interatomic exchange small. The second factor is a mutual compensation of the contributions from the valence band electrons.

\section{3. $L D A+U$ calculations}

For many systems containing $3 \mathrm{~d}$ transition atoms, the application of the LDA $+U$ scheme improves agreement with experiment over LDA calculations alone [23]. We performed LDA $+U$ calculations with $U$ equal to $0.1,0.2$ and $0.3 \mathrm{Ryd}$. To demonstrate the trends caused by the changing $U$ value, we show the Curie temperature and $J_{[220]}$ exchange, as well as the DOS in the middle and bottom panels of figures 2 and 3, respectively, for $U=0.1$ and 0.2 Ryd. The main effect of $U$ is the shift of the weight of the occupied Mn 3d spin-up states to lower energies. The changes in DOS distribution with the $U$ parameter are very small because the band gap is large, and hence the $3 \mathrm{~d} \uparrow$ states directly adjacent to the Fermi level are only slightly shifted towards the bottom of the valence band, whereas the $3 \mathrm{~d} \downarrow$ states are not changed at all. On the other hand, exchange parameter $J_{[220]}$ is found to be very sensitive to the value of $U$ (figure 3). In all cases, we obtain strong exchange splitting at the top of the valence band and very small interatomic exchange interaction for the nominal number of electrons. The LDA $+U$ calculations gave the following values for the Mn magnetic moment and the number of the $3 \mathrm{~d}$ electrons: $4.41 \mu_{\mathrm{B}}$ and 5.37 for $U=0.1 \mathrm{Ryd}, 4.43 \mu_{\mathrm{B}}$ and 5.39 for $U=0.2 \mathrm{Ryd}, 4.47 \mu_{\mathrm{B}}$ and 5.43 for $U=0.3$ Ryd. Below, we compare the calculated results with spectroscopic data.

The experimental $\mathrm{Mn}$ and $\mathrm{S} \mathrm{L}_{2,3}$ XES spectra of $\mathrm{Zn}_{0.9} \mathrm{Mn}_{0.1} \mathrm{~S}$ are compared to the calculated partial DOS ( $U=0.1 \mathrm{Ryd}$ ) in figure 4 . On the basis of other calculations for Mn-containing insulators and semiconductors, a $U$ value in this range is more physical than a straight LDA calculation for reproducing the correct energy positioning of localized Mn $3 \mathrm{~d}$ states. This is seen in the top panel of figure 4, where a superposition of the calculated Mn $3 \mathrm{~d}$ DOS and the $\mathrm{L}_{2,3}$ (2p-3d transition) emission spectrum is shown. It must be emphasized that this comparison serves only to illustrate the relationships between the DOS and the XES; the calculation is not intended to simulate the XES spectra. The dipole matrix elements, which would have redistributed the intensities throughout the DOS, were not included. As it is not intended to be a spectral simulation, we have included only the unbroadened DOS in the figure. Moreover, the calculation corresponds to zero temperature whereas the spectra were measured at room temperature.

In order to compare the spectroscopic measurements of the DOS to the calculations, the binding energies of the Mn and S 2p core holes (639.8 eV and $162.2 \mathrm{eV}$, respectively) [24, 25] were subtracted from the measured emission energy. The Mn spectrum and Mn 3d DOS (top panel) are located near the top of the valence band, in the same region as the S 3p DOS (middle panel). The Mn 4s DOS is not included in the comparison, as it does not generally make a significant contribution to the $\mathrm{L}_{2,3}$ XES, despite being involved in dipole allowed transitions. The feature $A$ that was indicated in figure 1 is also shown in figure 4 , and it is clear that the $\mathrm{S}$ $3 \mathrm{p}$ DOS suggests that this feature is, as expected, associated with hybridized $\mathrm{Mn} 3 \mathrm{~d} / \mathrm{S} 3 \mathrm{p}$ states. The S 3p DOS is not strongly reflected in the measured $\mathrm{S} \mathrm{L}_{2,3}$ XES spectrum (middle panel) because transitions from these levels are forbidden by dipole selection rules. The intensity in this region is due in part to the small amount of S 3s DOS in this region (resulting from $\mathrm{S} s p$ hybridization), as well as the partial d-type symmetry of the hybrid states. The $\mathrm{S}_{2,3}$ spectra of the $x=0.2,0.3$ samples are indistinguishable from that of the $x=0.1$ sample, and are therefore not included.

The main peak of the $\mathrm{S}_{2,3}$ XES is well represented by the $\mathrm{S} 3$ s DOS. Our calculations show that the two features seen near -8 and $-7 \mathrm{eV}$ are related to the $\mathrm{Zn} 3 \mathrm{~d}$ states, which our calculations reproduce at these energies. The $\mathrm{Zn}$ site DOS are not shown because there is no 


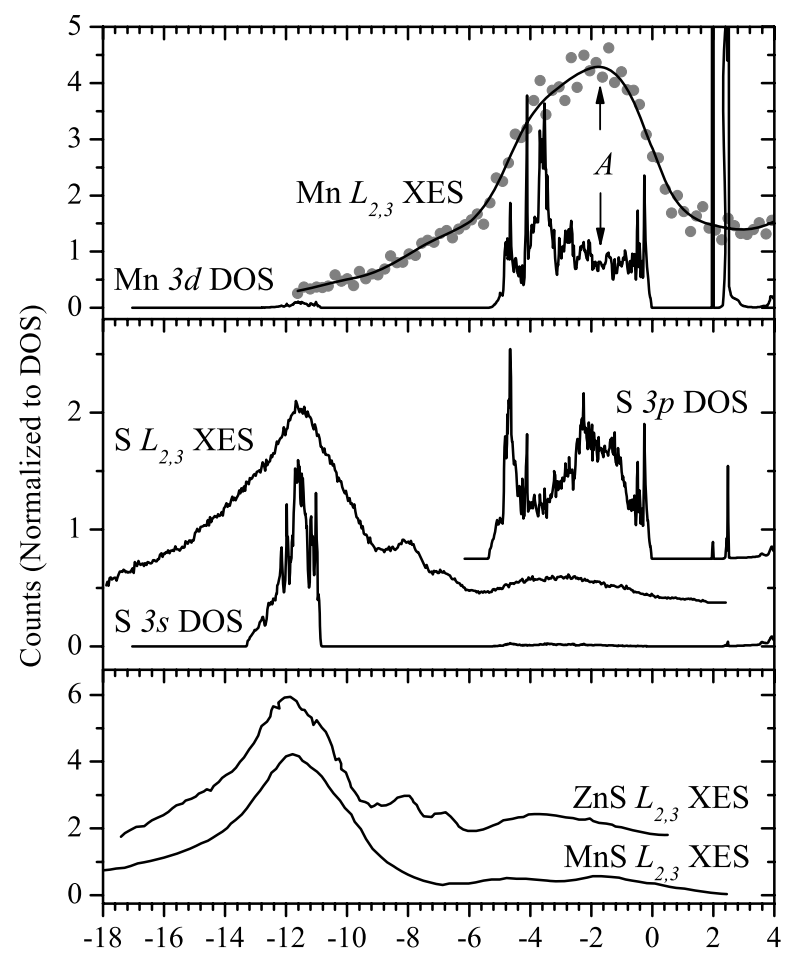

[Emission Energy - Binding Energy] (eV)

Figure 4. $\mathrm{Mn} \mathrm{L}_{2,3} \mathrm{XES}$, and $\mathrm{S} \mathrm{L}_{2,3} \mathrm{XES}$ of $\mathrm{Zn}_{0.9} \mathrm{Mn}_{0.1} \mathrm{~S}$ compared with calculated partial DOS, and $\mathrm{S} \mathrm{L}_{2,3} \mathrm{XES}$ of reference compounds $\mathrm{ZnS}$ and $\mathrm{MnS}$.

emission spectrum to compare with. Still, the $\mathrm{Zn} 3 \mathrm{~d} / \mathrm{S}$ 3s hybridization makes these features visible in the $\mathrm{S} \mathrm{L}_{2,3}$ spectra of doped crystals, as well as in the spectrum of the reference sample $\mathrm{ZnS}[26]$.

\section{Conclusions}

A study of the electronic structure of a series of dilute magnetic semiconductors $\mathrm{Zn}_{1-x} \mathrm{Mn}_{x} \mathrm{~S}$ was undertaken using soft $\mathrm{x}$-ray emission measurements and DFT calculations. Measurements of the $\mathrm{Mn} \mathrm{L}_{2,3}$ XES spectra suggest that clustering of the Mn impurities does not occur in these samples, in accordance with the suggestions of previous studies. A concentrationdependent shift in the energy of a spectral feature is observed and is attributed to changes in the hybridization of the $\mathrm{Mn} 3 \mathrm{~d}_{5 / 2}$ states with the $\mathrm{S} 3 \mathrm{p}$ states. The comparison of the measured XES spectra with the partial DOS calculated via DFT supports this assessment. Variations of the intra-atomic correlation parameters in the calculations have a noticeable effect on the interatomic exchange parameter $J_{[220]}$ for the $\mathrm{Zn}_{1-x} \mathrm{Mn}_{x} \mathrm{~S}$ system. The calculations show evidence for high magnetic moments and a lack of magnetic ordering in the materials.

\section{Acknowledgments}

Funding by the Research Council of the President of the Russian Federation (Grant NSH4192.2006.2), the Russian Science Foundation for Basic Research (Project 05-02-16438), the 
Natural Sciences and Engineering Research Council of Canada (NSERC), and the Canada Research Chair Program is gratefully acknowledged.

\section{References}

[1] Ohno H, Shen A, Matsukura F, Oiwa A, Endo A, Katsumoto S and Iye Y 1996 Appl. Phys. Lett. 69 363-5

[2] Ohno H 1998 Science 281 951-6

[3] Gould C, Schmidt G, Richter G, Fiederling R, Grabs P and Molenkamp L W 2002 Appl. Surf. Sci. 190 395-402

[4] Furdyna J K 1988 J. Appl. Phys. 64 R29-64

[5] Durst A C, Bhatt R N and Wolff P A 2002 Phys. Rev. B 65235205

[6] Ferrand D et al 2001 Phys. Rev. B 6385201

[7] Schuler T, Stern R, McNorton R, Willoughby S, Maclaren J, Ederer D, Perez-Dieste V, Himpsel F, LopezRivera S and Callcott T 2005 Phys. Rev. B 72 (4)

[8] Sandratskii L M and Bruno P 2003 J. Phys.: Condens. Matter 15 L585-90

[9] Rader O et al 2004 Phys. Rev. B 69075202

[10] Pong W F, Mayanovic R A, Kao J K, Hsieh H H, Pieh J Y, Chang Y K, Kuo K C, Tseng P K and Lee J F 1997 Phys. Rev. B 55 7633-40

[11] Lawniczak-Jablonska K, Perera R C C, Underwood J H, Gullikson E M and Iwanowski R J 1997 Phys. Rev. B 55 10376-81

[12] Perez-Dieste V et al 2004 Phys. Rev. B 70085205

[13] Okabayashi J et al 2004 J. Appl. Phys. 95 3573-5

[14] Cho K et al 2001 Phys. Rev. B 63155203

[15] Lawniczak-Jablonska K, Iwanowski R J, Golacki Z, Traverse A, Pizzini S, Fontaine A, Winter I and Hormes J 1996 Phys. Rev. B 53 1119-28

[16] Pong W F, Mayanovic R A, Wu K T, Tseng P K, Bunker B A, Hiraya A and Watanabe M 1994 Phys. Rev. B 50 7371-7

[17] Wyckoff R W G 1963 Crystal Structures (New York: Wiley)

[18] Grebennikov V I 2002 Surface Investigations: X-ray, Synchrotron and Neutron Techniques vol 11, p 41

[19] Kurmaev E, Ankudinov A, Rehr J, Finkelstein L, Karimov P and Moewes A 2005 J. Electron Spectrosc. Relat. Phenom. 148 1-4

[20] Pong W F, Mayanovic R A, Bunker B A, Furdyna J K and Debska U 1990 Phys. Rev. B 41 8440-8

[21] Sapra S, Nanda J, Anand A, Bhat S and Sarma D 2003 J. Nanosci. Nanotechnol. $3392-400$

[22] Sandratskii L M 2003 Phys. Rev. B 68224432

[23] Anisimov V I, Aryasetiawan F and Lichtenstein A I 1997 J. Phys.: Condens. Matter 9 767-808

[24] Kovtyukhova N I, Buzaneva E V, Waraksa C C, Martin B R and Mallouk T E 2000 Chem. Mater. 12 383-9

[25] Lawniczak-Jablonska K, Golacki Z, Paszkowicz W, Masek J, Johansson L S and Heinonen M 1994 J. Phys.: Condens. Matter 6 3369-78

[26] Zhou L, Callcott T A, Jia J J, Ederer D L and Perera R 1997 Phys. Rev. B 55 5051-61 